\title{
Formación en habilidades comunicativas desde la perspectiva de residentes y tutores en medicina
}

\author{
Beatriz Molinuevo, Xavier Clèries, Amor Aradilla-Herrero, Maria Nolla-Domenjó
}

Introducción. En las últimas décadas se ha producido un cambio sustancial respecto a la consideración de las habilidades comunicativas en el currículo de medicina. Actualmente, se considera una competencia nuclear del profesional sanitario, susceptible de entrenamiento y mejora.

Objetivo. Identificar el nivel de formación y las necesidades percibidas de médicos especialistas en formación (MEF) y tutores respecto al aprendizaje de habilidades comunicativas, como primer paso para diseñar programas efectivos adaptados al contexto actual.

Sujetos y métodos. Participaron 131 residentes y 79 tutores, de 12 centros hospitalarios de Cataluña, que cumplimentaron vía online un cuestionario autoadministrado.

Resultados. Un $74 \%$ de residentes y un $67 \%$ de los tutores habían recibido formación en habilidades comunicativas. Residentes y tutores coincidieron en la valoración del área en la que la formación es adecuada (entrevista clínica) y en las áreas susceptibles de mejora (manejo de emociones, gestión de situaciones difíciles y comunicación con familiares y cuidadores) durante el programa de MEF. Ambos manifestaron una alta predisposición a participar en futuros programas de formación.

Conclusiones. Existen deficiencias en la formación en habilidades comunicativas de residentes y tutores de medicina. Uno de los retos es asegurar que los residentes, independientemente de la especialidad, adquieran un nivel óptimo de competencias comunicativas una vez finalizado el periodo de formación.

Palabras clave. Educación médica. Formación médica especializada. Habilidades comunicativas. Residente. Tutor.

Training in communication skills according to residents' and tutors" perspective in medicine

Introduction. During last decades there has been a substantial change from the consideration of communication skills in the curriculum of medicine. Currently, it is considered a core competency of health professionals; it can be trained and improved.

Aim. To identify the level of training and the perceived needs of residents and tutors about the learning of communication skills as a first step in designing effective programs adapted to the current context.

Subjects and methods. Participants were 131 residents and 79 tutors, from 12 hospitals in Catalonia, who completed a self administered questionnaire via online.

Results. $74 \%$ of residents and $67 \%$ of the tutors had received training in communication skills. Residents and tutors agreed on the valuation of the area in which the training was appropriate (clinical interview) and in areas for improvement (managing emotions, managing difficult situations, and communication with family and caregivers) during the training system of health care professionals. Both showed a high willingness to participate in future training programs.

Conclusions. There are deficiencies in communication skills training of residents and tutors of Medicine. One of the challenges is to ensure that residents, regardless of specialty, acquire an optimal level of communicative competence upon completion of the training period.

Key words. Communication skills. Medical education. Resident. Specialist medical training. Tutor.
Departamento de Psiquiatría y Medicina Legal; Facultad de Medicina; Universitat Autònoma de Barcelona (B. Molinuevo). Parc Sanitari Pere Virgili (X. Clèries). Unidad de Calidad Docente e Innovación Educativa; Escola Universitària d'Infermeria Gimbernat; Universitat Autònoma de Barcelona (A. Aradilla-Herrero. Fundació Doctor Robert (M. Nolla-Domenjó) Barcelona, España.

\section{Correspondencia:}

Dra. Beatriz Molinuevo Alonso. Unidad de Psicología Médica. Departamento de Psiquiatría y Medicina Legal. Facultad de Medicina. Universitat Autònoma de Barcelona. Campus de Bellaterra, s/n. E-08193 Bellaterra (Barcelona)

E-mail:

beatriz.molinuevo@uab.cat

Agradecimientos:

A los profesionales integrantes del Área de Comunicación en el Ámbito de la Salud del Institut $d^{\prime}$ Estudis de la Salut (Generalitat de Catalunya)-Fundació Doctor Robert (Universitat Autònoma de Barcelona), de manera especial a R. Garcia-Guasch, C, Llubià y G. Tolchinsky. A las unidades docentes del Hospital Universitari Germans Trias i Pujol y de Badalona Serveis Assistencials, y a los residentes en formación y tutores por su participación voluntaria en el estudio piloto. A la Red de Comisiones de Docencia y Asesores de Catalunya. A las unidades docentes de los centros que accedieron a participar en el estudio: Althaia Xarxa Assistencial de Manresa, Capio Hospital Universitari Sagrat Cor, Consorci Sanitari de Terrassa, Corporació Sanitària Parc Taulí de Sabadell, Hospital Clínic de Barcelona, Hospital Dr. Trueta de Girona, Hospital Esperit Sant de Santa Coloma de Gramenet, Hospital General de Granollers, Hospital Sant Jaume de Calella, Institut Pere Mata de Reus, Institut Universitari Dexeus y Serveis de Salut Integrals Baix Empordà. A los médicos en formación y a los tutores que han participado en el estudio. 
Conflicto de intereses: No declarado.

Competing interests: None declared.

(c) 2014 FEM

\section{Introducción}

En las últimas décadas se ha producido un cambio sustancial respecto a la consideración de las habilidades comunicativas en el currículo de medicina $[1,2]$. Actualmente, se considera una competencia nuclear del profesional sanitario, susceptible de entrenamiento y mejora [3]. Las diversas instituciones vinculadas a la formación de grado y posgrado de los futuros médicos están adecuando sus programas al nuevo escenario [4].

En nuestro país, la adaptación de los estudios de grado al Espacio Europeo de Educación Superior ha supuesto la modificación de los planes de estudios en medicina que ha permitido la incorporación de la formación de habilidades comunicativas [5,6]. Sin embargo, no todos los programas de residentes -médicos especialistas en formación (MEF) - contemplan la formación de habilidades comunicativas $y$, en los que se recoge, existe gran heterogeneidad respecto a contenidos, duración y metodologías de aprendizaje, entre otros aspectos [7-10]. Por ello, uno de los retos vigentes es asegurar que los residentes, independientemente de la especialidad, adquieran un nivel óptimo de competencias comunicativas una vez finalizado el periodo de formación [11].

Evaluar el nivel de conocimientos previos de que disponen los residentes, conocer su nivel de satisfacción con los programas de formación actuales y detectar las áreas susceptibles de mejora es de gran utilidad para el diseño de programas formativos efectivos en competencias comunicativas. Según nuestro conocimiento, las investigaciones realizadas, además de ser insuficientes, contemplan un único centro o tan sólo alguna especialidad médica, y la información se ha recogido a través de una o dos preguntas generales del tipo '¿recibes formación en habilidades comunicativas?' o ‘invertirías parte de tu tiempo en recibir más formación en habilidades comunicativas?' $[8,12]$.

La figura del tutor ejerce una influencia decisiva en la formación de residentes, ya que se convierte en un modelo de referencia para el futuro médico. Por ello, es de especial importancia que el tutor haya recibido formación en habilidades comunicativas y demuestre esas competencias en la relación con pacientes, familiares y otros profesionales, y que presente actitudes positivas hacia la importancia de su aprendizaje como elemento imprescindible para la profesión médica. En este sentido, cabe destacar la existencia de estudios que afirman que no todos los tutores han recibido formación en dichas habilidades [13] e incluso infravaloran la importancia de la relación médico-paciente, lo que se convierte en un potencial obstáculo para modelar competencias comunicativas [14].

Por ello, este trabajo se centra en evaluar el conocimiento y las necesidades de formación en habilidades comunicativas de residentes y tutores de especialidades en medicina. Los objetivos específicos son:

- Examinar el nivel de formación en habilidades comunicativas de residentes y tutores de la formación médica especializada.

- Analizar el grado de satisfacción con la formación en habilidades comunicativas durante el grado y la formación especializada.

- Detectar las áreas de mejora de la formación y valorar la disposición a participar en programas de formación sobre habilidades comunicativas.

\section{Sujetos y métodos}

Se elaboró un cuestionario autoadministrado, con formato diferente para residentes y tutores. Contenía cuatro apartados, que incluían aspectos sociodemográficos, académicos, profesionales y sobre la formación en habilidades comunicativas. Posteriormente, se facilitó una versión en papel a diversos profesionales expertos en el área de la comunicación para garantizar su comprensión y valorar la idoneidad de las preguntas en relación a los objetivos propuestos. Una vez recogidas las sugerencias y modificado el instrumento, según sus propuestas, se diseñó una versión electrónica para su administración a través de Internet, con la herramienta de encuestas online 'Encuestafacil.com'. Seguidamente, en el año 2011, se llevó a cabo un estudio piloto en un centro hospitalario para detectar la existencia de algún tipo de problema tecnológico o de comprensión. Para ello se creó un apartado de sugerencias al final del instrumento. La versión definitiva está disponible bajo petición.

Durante el mes de febrero de 2012 se presentó el proyecto a la Red de Comisiones de Docencia y Asesores de Cataluña. El estudio iba dirigido a residentes y tutores del programa de formación especializada, excepto aquellos pertenecientes a Medicina de Familia y Comunitaria (MFyC), dado que en esta especialidad la formación en habilidades de comunicación se contempla de forma sistemática casi desde su creación. La dirección de 12 centros hospitalarios confirmó su interés en participar.

Con el objeto de poder garantizar el anonimato y la protección de datos personales, se remitieron las instrucciones a las unidades docentes de los centros para que éstos fueran los encargados de enviar a los residentes y tutores el enlace para participar. 
Asimismo, cuando los participantes entraron en el aplicativo, se les indicó que su participación era voluntaria y anónima y que los datos sólo se analizarían con fines estadísticos. El trabajo de campo se realizó durante los meses de abril y mayo de 2012, coincidiendo con el final del periodo de residencia.

Las muestras finales estaban compuestas por 131 residentes con una edad media de 29,10 \pm 4,33 años (rango: 25-48 años) y 79 tutores con una edad media de 45,44 \pm 7,42 años (rango: 26-65 años). Los porcentajes de participación fueron del 14,9\% y $36,7 \%$, respectivamente. Como se puede observar en la tabla I, en la muestra de residentes hay mayor proporción de mujeres $(64,1 \%)$ y aproximadamente el $75 \%$ han nacido en España. Más de la mitad ha obtenido la titulación de medicina en Cataluña y alrededor del 25\% fuera de España. La participación de residentes de último año es baja y alrededor del $60 \%$ pertenecen a especialidades médicas.

En la muestra de tutores, la participación por sexos fue similar (55,7\% de mujeres), la procedencia era mayoritariamente española y cerca del $80 \%$ pertenecían a especialidades médicas (Tabla I). La media de años de ejercicio como tutores de residentes fue de $5,44 \pm 3,60$, y el número medio de residentes a su cargo, de $4,24 \pm 2,47$.

\section{Análisis estadístico}

Los datos se han analizado mediante el programa estadístico SPSS v. 19. Se han calculado las frecuencias y porcentajes de las variables de estudio y se ha utilizado el estadístico chi al cuadrado para analizar la relación entre variables.

\section{Resultados}

\section{Formación en habilidades comunicativas}

Como se aprecia en la tabla II, cerca del 75\% de residentes ha recibido formación en habilidades comunicativas antes o durante la formación especializada. Cuando se analizan los datos en función del año de residencia, se observa que el porcentaje de R3 y R4 que ha recibido formación tiende a ser superior al de R1 y R2. No obstante, la diferencia no llega a ser estadísticamente significativa $\left(\chi_{(3)}^{2}=4,86\right.$; $p>0,05)$. Tampoco se observan diferencias en función de la especialidad $\left(\chi_{(2)}^{2}=1,12 ; p>0,05\right)$.

Realizar 'cursos específicos' es la vía más habitual de recibir formación y, en este sentido, cerca de la mitad de participantes ha realizado algún curso. En este caso sí se detectan diferencias estadística-
Tabla I. Características sociodemográficas y académicas de las muestras de residentes y tutores.

\begin{tabular}{|c|c|c|c|}
\hline & & Residentes $(n=131)$ & Tutores $(n=79)$ \\
\hline \multicolumn{2}{|c|}{ Edad media (desviación estándar) } & 29,10 (4,33 años) & 45,44 (7,42 años) \\
\hline \multirow{2}{*}{ Sexo } & Mujer & $84(64,1 \%)$ & $44(55,7 \%)$ \\
\hline & Hombre & $47(35,9 \%)$ & $35(44,3 \%)$ \\
\hline \multirow{5}{*}{ País de nacimiento } & España & $96(73,3 \%)$ & $75(94,9 \%)$ \\
\hline & Otros países & $35(26,7 \%)$ & $4(5,1 \%)$ \\
\hline & Europa & $6(17,1 \%)$ & $2(0,5 \%)$ \\
\hline & América Central y del Sur & $28(80,0 \%)$ & $1(0,3 \%)$ \\
\hline & Asia & $1(2,9 \%)$ & $1(0,3 \%)$ \\
\hline \multirow{3}{*}{$\begin{array}{l}\text { Universidad de } \\
\text { obtención del título }\end{array}$} & Cataluña & $84(64,1 \%)$ & - \\
\hline & Resto de España & $15(11,5 \%)$ & - \\
\hline & Fuera de España & $32(24,4 \%)$ & - \\
\hline \multirow{5}{*}{ Año de residencia } & Primero & $39(29,8 \%)$ & - \\
\hline & Segundo & $36(27,5 \%)$ & - \\
\hline & Tercero & $22(16,8 \%)$ & - \\
\hline & Cuarto & $28(21,4 \%)$ & - \\
\hline & Quinto & $6(4,6 \%)$ & - \\
\hline \multirow{4}{*}{ Especialidad } & Médica & $75(57,3 \%)$ & $63(79,7 \%)$ \\
\hline & Médica-quirúrgica & $30(22,9 \%)$ & $6(7,6 \%)$ \\
\hline & Quirúrgica & $20(15,3 \%)$ & $6(7,6 \%)$ \\
\hline & Laboratorio & $6(4,6 \%)$ & $4(5,1 \%)$ \\
\hline
\end{tabular}

-: no aplicado.

mente significativas en función del año de residencia, siendo más frecuente en los últimos años $\left(\chi_{(3)}^{2}=\right.$ $10,17 ; p<0,05)$. En cuanto a la especialidad, las diferencias no llegan a ser significativas $\left(\chi^{2}{ }_{(2)}=5,00\right.$; $p=0,08)$. Es destacable que únicamente un $18,3 \%$ manifiesta haber cursado 'asignaturas obligatorias durante la licenciatura', y un 30,5\%, 'asignaturas optativas' sobre habilidades comunicativas.

En el caso de los tutores, un 67,1\% de los encuestados responde haber recibido formación en habilidades comunicativas (Tabla III). Destaca que la for- 
Tabla II. Formación recibida en habilidades comunicativas antes o durante la formación MIR (residentes).

\begin{tabular}{|c|c|c|c|c|c|c|c|c|c|}
\hline & & \multirow[b]{2}{*}{ Total } & \multicolumn{4}{|c|}{ Año de residencia } & \multicolumn{3}{|c|}{ Especialidad } \\
\hline & & & $\mathrm{R} 1$ & R2 & R3 & $\mathrm{R} 4^{\mathrm{a}}$ & M & $M-Q$ & Q \\
\hline \multicolumn{2}{|c|}{ Formación en habilidades comunicativas } & $97(74)$ & $26(66,7)$ & $25(69,4)$ & $20(90,9)$ & $26(76,5)$ & $57(76,0)$ & $21(70,0)$ & $13(65,0)$ \\
\hline \multirow{4}{*}{$\begin{array}{l}\text { Tipo de } \\
\text { formación }\end{array}$} & Asignaturas optativas durante la licenciatura & $40(30,5)$ & $10(25,6)$ & $13(36,1)$ & $7(31,8)$ & $10(29,4)$ & $22(29,3)$ & $13(43,3)$ & $4(20,0)$ \\
\hline & Cursos de doctorado & $1(0,8)$ & 0 & 0 & $1(4,5)$ & 0 & $1(1,3)$ & 0 & 0 \\
\hline & Cursos específicos & $62(47,3)$ & $15(38,5)$ & $14(38,9)$ & $17(77,3)$ & $16(47,1)$ & $40(53,3)$ & $9(30,0)$ & $8(40,0)$ \\
\hline & Talleres en congresos & $14(10,7)$ & $4(10,3)$ & $3(8,3)$ & $3(13,6)$ & $4(11,8)$ & $12(16,0)$ & $1(3,3)$ & $1(5,0)$ \\
\hline
\end{tabular}

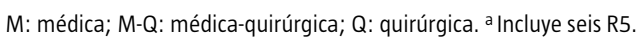

Tabla III. Formación recibida en habilidades comunicativas (tutores).

$n$ (\% sí)

\begin{tabular}{llc}
\hline & & $n(\%$ sí) \\
\hline Formación en habilidades comunicativas & $53(67,1)$ \\
\hline & \multicolumn{1}{l}{$\begin{array}{l}\text { Cursos específicos sobre entrevista } \\
\text { clínica y/o habilidades comunicativas }\end{array}$} & $35(66,0)$ \\
\cline { 2 - 3 } Tipo de & $\begin{array}{l}\text { Cursos de formación de formadores en } \\
\text { entrevista clínica y/o habilidades comunicativas }\end{array}$ & $26(49,1)$ \\
\cline { 2 - 3 } & \begin{tabular}{l} 
Talleres en congresos \\
\cline { 2 - 3 }
\end{tabular} & $4(7,5)$ \\
\cline { 2 - 3 } & Diplomas o másters & $3(5,7)$ \\
\cline { 2 - 3 } & Cursos de doctorado & 0 \\
\hline
\end{tabular}

mación de los tutores participantes ha sido fundamentalmente recibida en formato de cursos específicos sobre entrevista clínica o habilidades comunicativas y cursos de formación de formadores en dichas habilidades.

\section{Satisfacción con la formación en habilidades comunicativas}

En el cuestionario para residentes se preguntó por la satisfacción con la formación en diferentes áreas diferenciando estudios de licenciatura y programa de MEF. Como se observa en la tabla IV, la forma- ción en 'habilidades comunicativas de entrevista clínica' es el área mejor valorada durante la licenciatura, ya que el $60,5 \%$ se muestra satisfecho. Sin embargo, cerca del $40 \%$ manifiesta no sentirse satisfecho con la formación en 'habilidades comunicativas de manejo de emociones' y 'habilidades comunicativas en situaciones difíciles', y aproximadamente el 30\%, con la formación en 'comunicación con familiares y cuidadores' y 'comunicación con profesionales no sanitarios'.

En la tabla IV también se observan los datos correspondientes a la satisfacción en la formación de las habilidades comunicativas durante el programa de MEF. En general, se puede apreciar que existe mayor satisfacción que en la licenciatura en todas las áreas analizadas porque el porcentaje de residentes que se muestran satisfechos con la formación recibida supera el 50\%. El área de mayor satisfacción es la de "habilidades comunicativas en entrevista clínica' $(73,1 \%)$. Las áreas con mayor porcentaje de participantes insatisfechos son 'habilidades comunicativas en situaciones difíciles' y 'habilidades comunicativas de manejo de emociones' (alrededor del 15\%).

En el cuestionario destinado a los tutores se preguntó por la satisfacción con la formación en diferentes áreas durante el programa de MEF. Como se observa en la tabla $\mathrm{V}$, la valoración de los tutores es altamente coincidente con la de residentes respecto a las áreas de mayor y menor satisfacción. No obstante, es destacable que el grado de satisfacción tien- 
Tabla IV. Satisfacción de los residentes con la formación recibida en habilidades comunicativas.

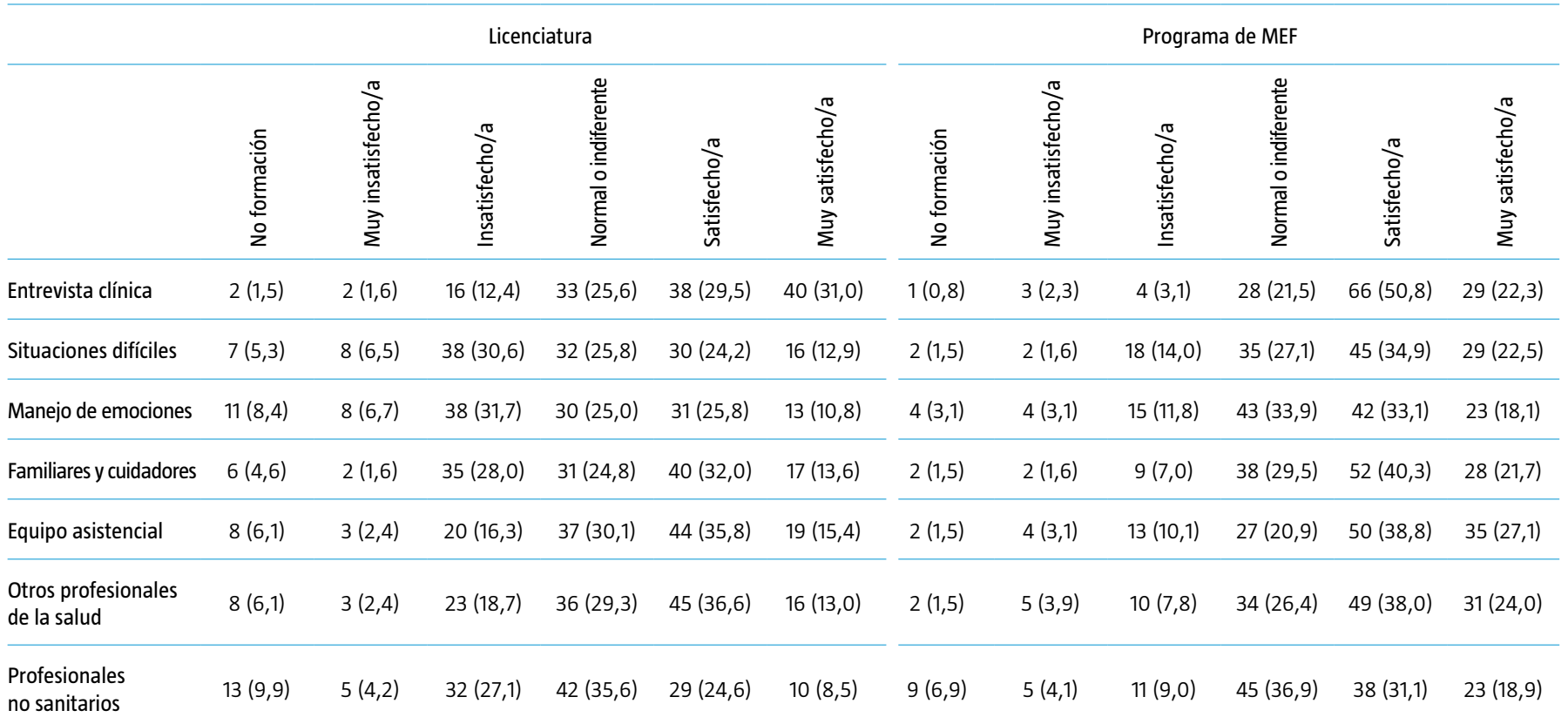

Entre paréntesis, porcentajes, que se han calculado sin tener en cuenta los participantes que no han recibido formación en cada área en particular. MEF: médico especialista en formación.

de a ser menor en todas las áreas, excepto en la de 'entrevista clínica'. Asimismo, más de un $20 \%$ de tutores se muestra insatisfecho, especialmente en el 'manejo de emociones' (41,8\%), la 'comunicación con profesionales no sanitarios' $(34,2 \%)$ y las 'situaciones difíciles' (31,7\%).

\section{Detección de áreas de mejora y participación} futura en programas de formación sobre habilidades comunicativas

Ante la pregunta sobre si consideran que 'el programa de formación de MEF debería dar más importancia a la formación en habilidades comunicativas', el $78,6 \%$ de residentes y el $84,8 \%$ de tutores respondieron afirmativamente.

Con posterioridad, se solicitó a los participantes que, para cada área de habilidades comunicativas considerada en el estudio, valoraran si se debería o no ampliar la formación. En general, en cada área, más del $50 \%$ de residentes y tutores consideran que debe ampliarse dicha formación. Es destacable que en las áreas 'situaciones difíciles', 'manejo de emociones' y 'comunicación con familiares y cuidadores' el porcentaje de residentes y tutores que muestran su acuerdo con mejorar la formación sea superior al $80 \%$ (Figura).
Tabla V. Satisfacción de los tutores con la formación que se facilita en habilidades comunicativas durante el programa de médico especialista en formación.

\begin{tabular}{|c|c|c|c|c|c|}
\hline & $\begin{array}{l}\text { Muy insa- } \\
\text { tisfecho/a }\end{array}$ & $\begin{array}{l}\text { Insatis- } \\
\text { fecho/a }\end{array}$ & $\begin{array}{l}\text { Normal o } \\
\text { indiferente }\end{array}$ & $\begin{array}{l}\text { Satis- } \\
\text { fecho/a }\end{array}$ & $\begin{array}{l}\text { Muy satis- } \\
\text { fecho/a }\end{array}$ \\
\hline & $n(\%)$ & $n(\%)$ & $n(\%)$ & $n(\%)$ & $n(\%)$ \\
\hline Entrevista clínica & 0 & $12(15,2)$ & $26(32,9)$ & $33(41,8)$ & $8(10,1)$ \\
\hline Situaciones difíciles & $4(5,1)$ & $21(26,6)$ & $28(35,4)$ & $22(27,8)$ & $4(5,1)$ \\
\hline Manejo de emociones & $6(7,6)$ & $27(34,2)$ & $23(29,1)$ & $21(26,6)$ & $2(2,5)$ \\
\hline $\begin{array}{l}\text { Comunicación con } \\
\text { familiares y cuidadores }\end{array}$ & $2(2,5)$ & $16(20,3)$ & $31(39,2)$ & $28(35,4)$ & $2(2,5)$ \\
\hline $\begin{array}{l}\text { Comunicación con miembros } \\
\text { del equipo asistencial }\end{array}$ & $1(1,3)$ & $15(19,0)$ & $29(36,7)$ & $30(38,0)$ & $4(5,1)$ \\
\hline $\begin{array}{l}\text { Comunicación con otros } \\
\text { profesionales de la salud }\end{array}$ & $1(1,3)$ & $15(19,0)$ & $35(44,3)$ & $26(32,9)$ & $2(2,5)$ \\
\hline $\begin{array}{l}\text { Comunicación con } \\
\text { profesionales no sanitarios }\end{array}$ & $3(3,8)$ & $24(30,4)$ & $36(45,6)$ & $15(19,0)$ & $1(1,3)$ \\
\hline
\end{tabular}

Finalmente, se preguntó a los participantes si estaban dispuestos a participar en programas futuros de formación en habilidades comunicativas. El 88,5\% 
Figura. Áreas en las que se debería ampliar la formación de habilidades comunicativas.

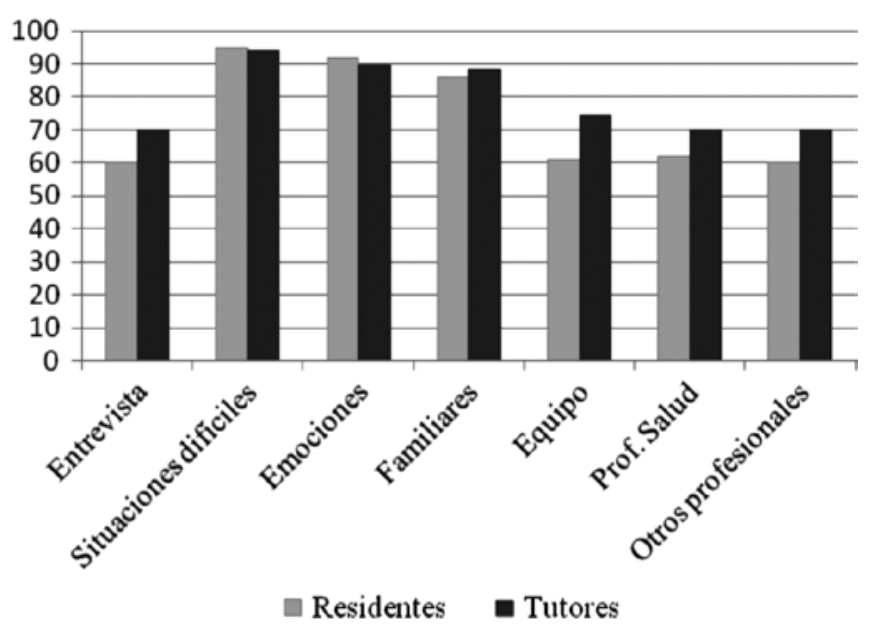

de residentes y el $87,3 \%$ de tutores respondieron afirmativamente.

\section{Discusión}

Según nuestro conocimiento, este es el primer trabajo que se realiza en nuestro país con el objetivo de evaluar el conocimiento y las necesidades de formación en habilidades comunicativas de residentes y tutores de especialidades médicas (con la excepción de la especialidad de MFyC). Los resultados indican que un alto porcentaje de residentes y tutores del programa de MEF pertenecientes a centros hospitalarios de la Red de Comisiones de Docencia y Asesores de Cataluña ha recibido formación en competencias comunicativas $(74 \%$ y $67,1 \%$, respectivamente) y que residentes y tutores coinciden en el área mejor valorada ('entrevista clínica') y las susceptibles de mejora ('manejo de emociones', 'situaciones difíciles' y 'comunicación con familiares y cuidadores'). Asimismo, residentes y tutores manifiestan una alta predisposición a participar en programas de formación futuros.

\section{Formación en habilidades comunicativas}

Este estudio permite afirmar que la mayor parte de los residentes y tutores participantes del programa actual de MEF han recibido formación en habilidades comunicativas, aunque existe un porcentaje significativo que indica no haber sido formado. No obstante, el porcentaje de residentes sin formación podría ser superior porque el nivel de participación es bajo y podrían haber participado los más motivados por los aspectos comunicativos de la práctica profesional. Como se ha comentado, la falta de estudios previos en nuestro país dificulta la comparación. En todo caso, destacan dos trabajos realizados con residentes de medicina interna en los que se pedía valorar la formación recibida a través de una escala Likert de cuatro puntos: 1 , nada; 2, poco; 3 , bastante, y 4, mucho [7,12]. En ambos estudios, los residentes percibían que su formación era insuficiente. En el estudio de Pujol y Nogueras [7] también se evaluó la percepción de los tutores y, a pesar de que era mejor que la de los residentes, seguía considerándose baja.

Aunque el presente trabajo no permite conocer los motivos exactos de la carencia de formación, una de las posibles explicaciones puede tener relación con haber cursado planes de estudios en los que esta competencia no se contemplaba, e incluso se entendía como una competencia innata y no susceptible de aprendizaje y mejora mediante estrategias formativas, es decir, no entrenable. El hecho de que el porcentaje de residentes que responde haber cursado asignaturas obligatorias sea inferior al $20 \%$ y el de quienes han cursado asignaturas optativas se sitúe en torno al 30\%, apoya en parte esta interpretación. Este resultado también puede deberse a una falta de reconocimiento de la importancia de la adquisición de competencias comunicativas para el ejercicio de la práctica clínica y que ello conlleve una baja participación en programas formativos relacionados. Otra posible explicación podría ser una escasa oferta formativa de cursos sobre competencias comunicativas, fuera del marco del programa de MEF.

\section{Satisfacción con la formación en habilidades comunicativas durante la licenciatura y el programa de MEF y detección de áreas de mejora}

Residentes y tutores coinciden a la hora de manifestar su nivel de satisfacción con la formación recibida en diferentes ámbitos del área de las habilidades comunicativas. Aunque no es posible discernir si la valoración se relaciona con el número de horas de formación, la calidad de ésta u otros aspectos, el área mejor evaluada es la de 'entrevista clínica', y las peor valoradas, las de 'manejo de emociones' y 'situaciones difíciles'. La inexistencia de estudios previos similares no permite establecer comparaciones.

El resultado sobre la alta satisfacción en el área de la entrevista clínica puede explicarse porque tradicionalmente la formación en competencias co- 
municativas se ha asociado sobre todo a habilidades relacionadas con la recogida de la historia clínica [15] y, por ello, es el área más trabajada en los planes de formación. Sin embargo, hoy día existe un amplio acuerdo en considerar que las habilidades comunicativas tienen relación también con otros aspectos relacionados con la comunicación, tanto dentro como fuera del encuentro médico-paciente, como es el caso de situaciones difíciles, manejo de emociones y comunicación con otras personas (familiares o cuidadores) o profesionales (equipo asistencial, otros profesionales de la salud u otros profesionales no sanitarios) [4].

Los resultados sobre insatisfacción con la formación y áreas de mejora ('manejo de emociones', 'situaciones difíciles' y 'comunicación con familiares y cuidadores') son relevantes fundamentalmente por diferentes motivos. En primer lugar, los dos informadores participantes en el estudio -residentes y tutores- son coincidentes en sus valoraciones a pesar de sus diferencias respecto al rol que desempeñan, el grado de responsabilidad clínica y el nivel de experiencia profesional. En segundo lugar, estas áreas coinciden con contenidos que no se han contemplado en el currículo tradicional del grado de medicina. En tercer lugar, los contenidos relacionados con estas áreas, además de ser insuficientes, no han contado con un espacio de formación propio y se han tendido a incluir dentro de cursos generales sobre entrevista clínica y habilidades comunicativas. Finalmente, durante la última década, expertos internacionales enfatizan la importancia de la formación en dichas áreas en los estudios de grado y posgrado $[1,2]$.

\section{Participación futura en programas de formación sobre habilidades comunicativas}

Ambos colectivos muestren una alta predisposición a participar en programas formativos futuros. Este resultado sugiere que las habilidades comunicativas son importantes para la competencia profesional y que son susceptibles de entrenamiento y mejora a lo largo de la trayectoria profesional. No obstante, como se ha indicado, la 'deseabilidad social' puede estar sesgando este resultado.

\section{Limitaciones}

Los resultados de este trabajo deben interpretarse con precaución tomando en consideración determinadas limitaciones. En primer lugar, cabe destacar una baja participación en el estudio, fundamentalmente de residentes (14,9\% de residentes). Aun- que los motivos de la baja participación pueden ser múltiples (p. ej., procedimiento de reclutamiento de la muestra), el momento de recogida de la información ha coincidido con una situación política, económica y social compleja. No obstante, el porcentaje de participación de tutores $(36,7 \%)$ es similar a estudios previos, en los que se sitúa en torno al $30 \%$, y en algunos casos, inferior [16]. En segundo lugar, aunque la generalización de resultados a la comunidad de residentes y tutores de especialidades en medicina de nuestro país se encuentre limitada, destaca que en el estudio participan 12 centros asistenciales.

\section{Implicaciones para la práctica}

Los datos sugieren que convendría asegurar que, una vez finalizado el programa de MEF, los futuros médicos hayan recibido entrenamiento en competencias comunicativas. Si las primeras relaciones que los residentes mantienen con los pacientes son inadecuadas, puede suponer una pérdida de confianza en las primeras etapas de formación y amenazar una atención centrada en el paciente [17]. Recientemente, se ha mostrado que el entrenamiento en habilidades comunicativas tiene efectos a largo plazo, sobre todo en relación a la percepción de autoeficacia [18].

Igualmente, sería pertinente que los tutores hubieran recibido formación antes de tutorizar residentes, ya que aunque no impartan módulos específicos sobre competencias comunicativas, sí son modelos de referencia para los residentes y, por lo tanto, uno de los 'agentes' más influyentes en su formación y en el modelaje de comportamientos deseables. La relación residente-tutor constituye el eje fundamental sobre el cual gira todo el proceso formativo y los tutores adquieren un importante papel como facilitadores y canalizadores del proceso de aprendizaje [14] .

La formación en habilidades comunicativas dentro del programa de MEF debería cubrir todas las áreas relacionadas con las competencias comunicativas para la práctica clínica, especialmente 'manejo de emociones', 'situaciones difíciles' y 'comunicación con familiares y cuidadores'. Se debería garantizar una formación en dichas áreas orientada a que los futuros especialistas puedan gestionar adecuadamente sus propias emociones, así como detectar señales emocionales y preocupaciones de los pacientes, y facilitar la verbalización de éstas [19]. Incluso se ha llegado a afirmar que es mejor que los médicos detecten incorrectamente las emociones de los pacientes que no detectarlas [20]. La adquisi- 
ción de habilidades comunicativas permite un correcto ejercicio de la profesión en condiciones saludables, especialmente por lo que se refiere al impacto psicoafectivo que comporta la tarea asistencial [21]. Por ejemplo, estudios previos destacan que una de las tareas comunicativas más difíciles de la tarea asistencial es dar malas noticias, ya que amenaza la gestión de las propias emociones y genera altos niveles de estrés [22].

\section{Futuras investigaciones}

Una vez cubiertas las necesidades formativas, futuras investigaciones deberían encaminarse a analizar el impacto de la formación en los niveles de satisfacción y de bienestar personal de pacientes (familiares) y profesionales, la precisión diagnóstica, la adhesión terapéutica y el uso optimizado de recursos, entre otros aspectos.

Bibliografía

1. Makoul G. Essential elements of communication in medical encounters: the Kalamazoo consensus statement. Acad Med 2001; 76: 390-3.

2. General Medical Council. Tomorrow's Doctors. Outcomes and standards for undergraduate medical education. London: GMC; 2009

3. Borrell C, Epstein R. The International Conference on Communication and Health of Barcelona 2000. One more step in the right direction. Aten Primaria 2001; 27: 449-51.

4. Von Fragstein M, Silverman J, Cushing A, Quilligan S, Salisbury H, Wiskin C. UK consensus statement on the content of communication curricula in undergraduate medical education. Med Educ 2008; 42: 1100-7.

5. Borrell-Carrió $\mathrm{F}$, Clèries $\mathrm{X}$, Paredes-Zapata $\mathrm{D}$, Borrás-Andrés JM, Sans-Corrales M, Mascort-Roca JJ. Proceso de Bolonia (VI): aprendiendo comunicación para la salud en el Grado de Medicina. Educ Med 2012; 15: 197-201.

6. Molinuevo B, Escorihuela RM, Fernández-Teruel A, Tobeña A Torrubia R. How we train undergraduate medical students in decoding patients' nonverbal clues. Med Teach 2011; 33: 804-7.
7. Pujol R, Nogueras A. La formación de los médicos residentes de medicina interna. A propósito de una encuesta de opinión. Med Clin (Barc) 2002; 118: 545-50.

8. López F, Moro J, Tejedor J. La calidad percibida en la formación por los residentes de anestesia y reanimación. Rev Esp Anestesiol Reanim 2007; 54: 340-8.

9. Moore P, Gómez G, Kurtz S. Doctor-patient communication: one of the basic competencies, but different. Aten Prim 2012; 44: 358-65.

10. Millán J, Gutiérrez-Fuentes JA. 'Enseñar a ser médicos': un análisis de opinión de los médicos implicados en la docencia de la clínica práctica (I). Conclusiones del análisis cualitativo y metodología para un estudio cuantitativo. Educ Med 2012; 15: 143-7.

11. Palés J, Gual A. Medical education in Spain: current status and new challenges. Med Teach 2008; 30: 365-9.

12. Meijide H, Míguez RR, De la Iglesia F. La formación de médicos internos residentes de medicina interna en Galicia: encuesta de opinión. Galicia Clin 2010; 71: 153-9.

13. Perron NJ, Sommer J, Hudelson P, Demaurex F, Luthy C, Louis-Simonet M, et al. Clinical supervisors' perceived needs for teaching communication skills in clinical practice. Med Teach 2009; 31: e316-22.

14. Côté $\mathrm{L}$, Leclère $\mathrm{H}$. How clinical teachers perceive the doctorpatient relationship and themselves as role models. Acad Med 2000; 75: 1117-24.

15. Wouda JC, Van de Wiel HB. How to attain expertise in clinical communication? Paediatr Respir Rev 2013; 14: 213-8.

16. Pedregal M, Molina E, Morón A, Freire P, Pardo J, Mora F. Formación de los tutores de residentes de Andalucía en entrevista clínica y utilización de técnicas de consulta. Tribuna Docente 2007; 9: 1-8.

17. Stewart MA. Effective physician-patient communication and health outcomes: a review. CMAJ 1995; 152: 1423-33.

18. Gulbrandsen P, Jensen BF, Finset A, Blanch-Hartigan D. Long-term effect of communication training on the relationship between physicians' self-efficacy and performance. Patient Educ Couns 2013; 91: 180-5.

19. Zimmermann C, Del Piccolo L, Finset A. Cues and concerns by patients in medical consultations: a literature review. Psychol Bull 2007; 133: 438-63.

20. Blanch-Hartigan D. Patient satisfaction with physician errors in detecting and identifying patient emotion cues. Patient Educ Couns 2013; 93: 56-62.

21. Dobkin PL, Hutchinson TA. Primary prevention for future doctors: promoting well-being in trainees. Med Educ 2010; 44: 224-6.

22. Shaw J, Brown R, Heinrich P, Dunn S. Doctors' experience of stress during simulated bad news consultations. Patient Educ Couns 2013; 93: 203-8. 